\title{
Ethics and innovation in medicine
}

George J Agich Cleveland Clinic Foundation, Cleveland, Ohio, USA

How should one think about innovation in medicine and surgery? Increasingly, the answer to this question has involved reference to what might be called the regulatory ethics paradigm (REP). The regulatory ethics paradigm holds that deviations from standard care involve a degree or kind of experimentation that requires the application of a set of procedures designed to assure the protection of the rights and welfare of the subjects of research

In REP, innovative treatments are regarded as questionable until they are framed in a research protocol with formal mechanisms of informed consent. The protocol must be reviewed and approved by an Institutional Review Board (IRB) or equivalent. The regulatory ethics paradigm in effect imposes the condition that clinical innovations be conducted according to scientific research methodologies. It creates the presumption that without review by an IRB, innovation cannot be conducted in an ethically defensible fashion. The regulatory ethics paradigm also requires the preparation of investigational protocols according to sound evidentiary and methodological standards. In so doing, it creates a presumption that innovations that are not rigorously validated are ethically dubious. These assumptions have deep roots.

The Belmont Report, for example, articulated the orienting intuition that "radically new procedures ... should . . . be made the object of formal research at an early stage [emphasis added] in order to determine whether they are safe and effective". The Belmont Report takes the view that formal research to establish safety and efficacy of new interventions is usually feasible at an early stage in the development of a novel intervention. It thus establishes a bias that innovative treatments be conducted and evaluated under a research protocol that has passed muster at an IRB review.

Given the historical and social context of the movement to protect the subjects of research, it is not surprising that REP tends to assimilate activities that share a family resemblance with scientific research. Clinical experimentation understandably was regarded as sufficiently research-like that its participants should be afforded the protections of REP. The Belmont Report, however, offered no deep analysis of experimentation in the context of patient care or the development of novel treatments.

The regulatory ethics paradigm tends to regard innovative treatment simply as a departure from standard and accepted treatment alternatives. It thus overlooks those clinical situations or fields of medicine in which accepted treatments are ineffective or burdensome. To be sure, some of the core documents forming REP have included an awareness of the need for using unproven procedures, but there has not been much elaboration of the boundaries among research, innovation, and practice.

The recent revision of the Declaration of Helsinki importantly recognises that in the treatment of a patient, where proven prophylactic, diagnostic and therapeutic methods do not exist or have been ineffective, the physician, with informed consent from the patient, must be free to use unproven or new prophylactic, diagnostic and therapeutic measures, if in the physician's judgment they offer hope of saving life, re-establishing health or alleviating suffering (paragraph 30 ). ${ }^{2}$

Such statements appropriately point to a land outside the pale of REP. This borderland, however, is largely underdeveloped regarding the procedures and standards that should apply. Given the widespread acceptance of REP, bioethicists have not been inclined to examine closely the actual processes by which clinical innovations are developed and the initial conditions prerequisite for the application of the paradigm. This omission is important, because it has permitted the extension of REP into the domain of clinical innovation without an adequate assessment of the advantages and disadvantages of such an expansion.

The formal scientific research that REP envisions simply assumes that a degree of standardisation of device, technique, or procedure exists. Before a scientific research protocol is feasible, however, a developmental process must occur. In some cases, a significant and complex developmental effort is required to bring a procedure to the point at which formal research is possible. In the case of complex surgery, such as coronary artery bypass graft surgery (CABG), ${ }^{3-4}$ the procedures evolved against a background of no or very poor therapeutic options, underwent technical alterations as they were employed, and were dependent upon analogous advances in imaging, anaesthesia, and postoperative care. The developmental effort involves, among other matters, identifying or clarifying the types of patients who might benefit most from the new intervention, standardising the process or procedures used, and resolving technical problems in 
the application of the procedure. The regulatory ethics paradigm insists that such matters be reviewed by an IRB, but the structure of review typical of REP cannot effectively monitor the complex processes involved. The typical REP review is conducted by a committee relying only on the submitted application, protocol, and informed consent documents. The complex processes characteristic of clinical innovation are often not reducible to a scientific protocol. They typically involve intuition, experience, and an evolving knowledge about the treatment and disease processes and the interaction between treatment and pathology. While scientific knowledge and judgment are certainly involved, the formal methodology required in a scientific protocol is often not attainable or not useful in the early phases of development.

Clinical protocols addressing practical clinical concerns and endpoints rather than scientific hypotheses or methods are often used instead. Such protocols frequently undergo revision as experience accrues, so they evolve over time and present a moving target for the REP review process. The protocols articulate clinical goals and incorporate the complex processes of discovery and accrued experience with a developing procedure. They can be remarkably unlike scientific protocols that proceed from a clear statement of a research question under the (relatively) rigid constraints of scientific methodology. Innovation tolerates, even thrives on, intuition, experience, and uncertainty whereas scientific research proceeds under methodological constraints from a base of established knowledge toward its goal of hypothesis confirmation or disconfirmation.

The demand that a clinical trial be undertaken in a field of medicine undergoing rapid and dynamic development can actually thwart innovation. Under some circumstances, delay in the development and adoption of any new treatment is a price worth paying. Whenever there are other standard treatments that have an acceptable degree of success and are not burdensome, clinical trials are justified It is less clear that delays are ethically justified in fields of medicine or diseases where there are no satisfactory treatments or where the treatments are burdensome or otherwise problematic. Criteria as vague as "burdensome" or "problematic" are, of course, fraught with controversy and open to dispute.

The suggestion that clinical innovations might be treated otherwise than under REP is likely to be met with the strenuous objection that the rights and welfare of the subjects of research require the protection afforded by REP. Such a response has increasingly brought activities such as quality improvement and technical departures from standard care under the watchful eye of IRBs, but it should be obvious that the response begs the question. More importantly, the response does not address the deeper question about the legitimate place of innovation in the clinical practice of medicine and the ethical standards that should apply.

If some forms of innovation in medical care are not properly regarded as research, then the research protections that REP provides are not necessary or appropriate. Saying this in no way diminishes the concern over the rights and welfare of the patients undergoing the novel interventions. We need to seriously consider that REP may not represent the best statement of the ethical standards appropriate for innovative care.

The question is not whether patients accepting innovative interventions should be accorded informed consent or whether their welfare should be protected, but only whether the formal REP requirements provide the only legitimate means to accomplish the ethical objectives. Coupled with a preference for formal clinical trials by academic medicine and regulatory bodies, REP has lent an authoritative ethical justification to the call for validating new treatments in clinical trials. This ideal, however, has established the presumption that innovations in the treatment of patients should occur only as part of a formal research protocol, preferably in a clinical trial. Such a presumption can be counterproductive.

It is time for bioethicists to think innovatively about the ethical problems associated with innovation in patient care. To do so, bioethics will need to pay closer attention to the actual clinical, institutional, and professional processes that operate in the development of novel therapeutic interventions. Inevitably, they will need to question the accepted truths of REP, a task that will not be easy.

George F Agich, PhD, is the F F O'Neill Chair in Clinical Bioethics and Chairman, Department of Bioethics, Foint Appointment, Transplant Center, Cleveland Clinic Foundation, Cleveland, Ohio, USA.

\section{References}

1 National Commission. The Belmont report: ethical principles and guidelines for the protection of human subjects of research. Washington DC: US Government Printing Office, 1979.

2 World Medical Association. World Medical Association Declaration of Helsinki: ethical principles for medical research involving human subjects. Fournal of the American Medical Association 2000;284:3043-5.

3 Favaloro RG. Critical analysis of coronary artery bypass graft surgery: a 30-year journey. Fournal of the American College of Cardiology 1998;31 (suppl B):1-63B.

4 Favaloro RG. Landmarks in the development of coronary artery bypass surgery. Circulation 1998;98:466-78. 